\title{
Inactivación Térmica de Pectinmetilesterasa en Jugo de Uchuva (Physalis peruviana L.)
}

\author{
Yamid A. Pinchao(1), Oswaldo Osorio(2)* y Diego Mejía(3) \\ Universidad de Nariño. (1) Ingeniero Agroindustrial, Investigador departamento procesos industriales. $\left(2^{*}, 3\right)$ \\ Docentes Investigadores Facultad de Ingeniería Agroindustrial, Departamento procesos industriales Ciudad \\ Universitaria Torobajo - Calle 18 Cr 50, San Juan de Pasto - Colombia (e-mail: yamidalexis@hotmail.com; \\ osorio_oswaldo@hotmail.com; diegomejiaes@udenar.edu.co)
}

${ }^{*}$ Autor a quien debe dirigirse la correspondencia

Recibido Ene. 16, 2014; Aceptado Feb. 19, 2014; Versión final recibida Abr. 7, 2014

\section{Resumen}

Se analiza la posibilidad de procesamiento industrial de la uchuva (Physalis peruviana L.). Para ello se estudió el efecto de la pasteurización sobre la reducción en la actividad de la pectinmetilesterasa y sobre las características sensoriales del jugo de uchuva. Se aplicaron dos diseños experimentales para el proceso térmico: el primero con temperaturas entre 60 y $90^{\circ} \mathrm{C}$ y tiempos de exposición entre 20 y $60 \mathrm{~s}$ y el segundo con temperaturas entre 70 y $80^{\circ} \mathrm{C}$ y tiempos entre 10 y $30 \mathrm{~s}$. El jugo fue pasteurizado en tubos capilares para micro hematocrito. La actividad enzimática residual fue evaluada mediante espectrofotometría UV-VIS a $620 \mathrm{~nm}$. Las propiedades sensoriales (acidez, color, olor y sabor), se avaluaron mediante panel entrenado $(n=15)$. Como resultado, el punto óptimo de pasteurización se encontró a una temperatura de $80^{\circ} \mathrm{C}$ por $10 \mathrm{~s}$ con una actividad enzimática residual del $9,32 \%$. Con estos tratamientos térmicos no se apreciaron cambios sensoriales significativos $(P>0.05)$ entre el jugo pasteurizado y el jugo natural.

Palabras claves: pasteurización, pectinmetilesterasa, capilares, espectrofotometría, uchuva.

\section{Thermal Inactivation of Pectinmethylesterase in Cape Gooseberry Juice (Physalis peruviana L.)}

\begin{abstract}
The possibility of industrial processing of cape gooseberry (Physalis peruviana L.) is analyzed. To do this, the effect of pasteurization on reduction in pectinmethylesterase activity and sensory characteristics of gooseberry juice was determined. Two experimental designs were applied to the thermal process: the first with temperatures between $60^{\circ} \mathrm{C}$ and $90^{\circ} \mathrm{C}$ and exposure times between $20 \mathrm{~s}$ and $60 \mathrm{~s}$ and the second with temperatures between $70^{\circ} \mathrm{C}$ and $80^{\circ} \mathrm{C}$ and times between $10 \mathrm{~s}$ and $30 \mathrm{~s}$. The juice was pasteurized in micro hematocrit capillary tubes. The residual enzyme activity was evaluated by spectrophotometry at 620 $\mathrm{nm}$ UV-VIS. The sensory properties such as acidity, color, smell and taste, were valued by trained panel $(\mathrm{n}$ $=15$ ). As a result the optimum pasteurization was found at a temperature of $80^{\circ} \mathrm{C}$ for $10 \mathrm{~s}$ with a residual enzyme activity of $9.32 \%$. With these heat treatments, no significant sensory changes $(P>0.05)$ were observed between pasteurized juice and natural juice.
\end{abstract}

Keywords: pasteurization, pectinmethylesterase, hair, spectrophotometry, uchuva. 


\section{INTRODUCCIÓN}

La uchuva (Physalis peruviana L.) es originaria de los Andes Suramericanos principalmente de Perú, Colombia y Ecuador (Mendoza, 2012), el autor menciona que la uchuva colombiana posee un potencial económico muy importante en el mercado internacional debido a que Colombia es el primer productor mundial de uchuva, seguido por Sudáfrica. Algunos autores han reportado que actualmente se cultivan tres tipos de uchuva originarias de Colombia, Kenia y Sudáfrica, pero la uchuva colombiana se caracteriza por tener una mejor coloración y mayor contenido de azúcares, características que la hacen más apetecible en los mercados (Mendoza, 2012; Fischer et al., 2005).

Las frutas constituyen un grupo de alimentos necesarios para la salud y bienestar, sus derivados como son los jugos, se han convertido en un componente básico en cualquier dieta sana y equilibrada (Vila, 2006), es así que para el caso de la uchuva, existe la posibilidad de generar un mercado a nivel internacional debido a su carácter exótico-nutricional y al cambio en los hábitos alimenticios de la población, además esta fruta posee características tanto fisicoquímicas como organolépticas que permiten obtener diversos productos transformados, como son el contenido en pulpa (70\%), los sólidos solubles (14\%), el pH alrededor de 3.4 y su color, aroma y sabor son parámetros que, favorecen su aprovechamiento industrial (García et al., 2008).

Un problema común asociado a los jugos de fruta es la pérdida de turbidez, consistencia, separación de fases, decoloración y gelificación, provocada por enzimas pécticas, particularmente la enzima pectinmetilesterasa (PME), (Basak et al., 1996; Carbonell et al., 2006). La PME (EC 3.1.1.11) se presenta de forma natural en plantas, ataca los grupos carboxilo de la pectina presente en la pared celular de los frutos, Hidroliza los enlaces éster de la pectina presente en los jugos de fruta provocando la pérdida de turbidez que es atribuida a la suspensión de partículas como proteínas, pectinas, lípidos, hemicelulosas, celulosas y otros componentes minoritarios (Baker y Cameron, 1999). La PME provoca cambios importantes en las sustancias pécticas, carbohidratos, ácidos orgánicos, compuestos fenólicos y otros componentes que afectan las características sensoriales como color, aroma, sabor y textura, afectando de alguna manera el procesamiento de esta importante fruta de los andes tropicales (Willats et al., 2001).

La presencia de PME provoca que en las bebidas de estos frutos se formen dos fases, una de ellas clara en la parte superior y otra turbia en la parte inferior dejando un aspecto visible indeseado en el producto final; sin embargo, para reducir la actividad PME en alimentos con alto contenido de pectina una alternativa es la pasteurización (Cinquanta et al., 2010; Anthon et al., 2002), con la cual, se logra reducir a niveles aceptables $\leq 10 \%$ la actividad enzimática residual, factor problema en la calidad de jugos (Osorio et al., 2008; Carbonell et al., 2006), sin embargo los jugos comerciales conservados por pasteurización sometidos a temperaturas altas y tiempos prolongados, reducen también componentes deseables como nutrientes, color, aroma y textura que se destruyen en diferentes porcentajes, resultando en pérdidas significativas de calidad (Tribess y Tadini, 2006; Raviyan et al., 2005), de ahí la importancia de optimizar el tratamiento térmico aplicado mediante la aplicación de diseños experimentales, que permitan conservar al máximo los atributos de calidad del jugo (Montgomery y Runger, 2003). Aspectos técnicos que aún no han sido abordados en la Uchuva y que de alguna manera potencializarían la posible industrialización de este importante fruto de las zonas andinas, donde el fruto se produce. Con estos antecedentes presentados el objetivo de este trabajo fue investigar los efectos de la pasteurización sobre la actividad residual de la enzima PME y sobre las características sensoriales del jugo de uchuva (Physalis peruviana L.).

\section{MATERIALES Y MÉTODOS}

\section{Obtención de la muestra}

La uchuva fue obtenida de la zona Andina Colombiana, municipio de Funes, Vereda el Chapal, departamento de Nariño y procesada en el laboratorio de investigación en conservación y calidad de alimentos de la Facultad de Ingeniería Agroindustrial (Universidad de Nariño) sede Torobajo Pasto (Nariño), localizado a $2527 \mathrm{msnm}$., con temperatura promedio de $14{ }^{\circ} \mathrm{C}$ y una humedad relativa de $70 \%$.

\section{Extracción de jugo}

Los frutos se seleccionaron y clasificaron de acuerdo al estado de maduración No. 4 según la norma Colombiana ICONTEC NTC 4580; se lavaron y desinfectaron por inmersión en una solución de hipoclorito de sodio (50 ppm) durante 5 minutos, lavándolas posteriormente con agua potable. El jugo fue extraído usando una despulpadora de frutas en acero inoxidable marca JAVAR DF-300 de la Unidad de Producción Agroindustrial de la Universidad de Nariño, el jugo se llevó a congelación a $-23{ }^{\circ} \mathrm{C}$, para posteriores análisis. 


\section{Diseño experimental}

El proceso de pasteurización para inactivación enzimática se optimizo mediante la aplicación de dos diseños experimentales, las condiciones de pasteurización, se establecieron, con base a estudios realizados en jugos de naranja, (Navarro et al., 2011), en tomate de árbol (Maca et al., 2012), Fresa (Osorio et al.,2008) planteando un diseño factorial multinivel, con temperaturas desde los $60^{\circ} \mathrm{C}$ hasta los $90^{\circ} \mathrm{C}$ a 4 niveles, con tiempos de exposición de $20 \mathrm{~s}$ a $60 \mathrm{~s}$ a 3 niveles y como única variable respuesta la actividad enzimática residual PME.

Posteriormente se desarrolló un diseño de composición central y puntos estrella con superficie de respuesta para optimizar la combinación temperatura-tiempo de inactivación de la enzima PME, este diseño tiene como objetivo alcanzar un porcentaje igual ó menor al 10\% de Actividad Residual PME, con temperaturas desde $70^{\circ} \mathrm{C}$ hasta $80^{\circ} \mathrm{C}$ a 3 niveles y tiempos de exposición desde $10 \mathrm{~s}$ hasta $30 \mathrm{~s}$ a 3 niveles, con 5 puntos centrales, 2 puntos axiales para temperatura, 2 puntos axiales para tiempo y como variable respuesta la actividad residual. Los dos diseños fueron aleatorizados y corridos con la matriz principal más dos repeticiones. Los datos obtenidos se analizaron con el paquete estadístico Statgraphics Centurion XVI.II, aplicando un análisis de varianza mediante Tabla Anova, Superficie de Respuesta y Óptima Respuesta, todo con un nivel de confianza del 95\% (Miller y Miller, 2002; Montgomery y Runger, 2003).

Una vez establecido el punto óptimo de pasteurización (Diseño de Composición Central) y según la información reportada para análisis sensorial de alimentos de Anzaldúa (1994), el jugo de uchuva se sometió a combinaciones de pasteurización de $70^{\circ} \mathrm{C} \times 10 \mathrm{~s}, 80^{\circ} \mathrm{C} \times 10 \mathrm{~s}, 90^{\circ} \mathrm{C} \times 10 \mathrm{~s}$ y se utilizó una muestra de jugo sin pasteurizar como referencia para realizar el análisis sensorial (Miller y Miller, 2002).

\section{Tratamiento térmico}

La pasteurización para inactivación enzimática se aplicó bajo la metodología desarrollada por Anthon et al. (2002), colocando cada muestra de jugo en tubos capilares de vidrio para microhematocrito $(1.5 \times 100 \mathrm{~mm}$, Daslab®) con capacidad de $100 \mu \mathrm{L}$, los tubos se colocaron en un baño termostatado (EYELA N 1001S-WD $115 \mathrm{~V}$ ) y se sometieron a las temperaturas y tiempos de cada diseño experimental, la temperatura se midió con un lector de temperatura portátil (TECPEL DTM-305), al cual se acopló una termocupla tipo K. Posterior al calentamiento los tubos se trasladaron a un baño de agua con hielo a $5{ }^{\circ} \mathrm{C}$ a fin de lograr el choque térmico en el jugo, consecutivamente las muestras fueron almacenados para los análisis de actividad enzimática.

Para conocer si el punto óptimo de pasteurización encontrado, afecto las características sensoriales se procedió a pasteurizar las muestras de jugo en un pasteurizador construido en el laboratorio de investigación en conservación y calidad de alimentos de la Universidad de Nariño, utilizando un baño termostático (Eyela OSB 2000), con precisión de $\pm 0,1^{\circ} \mathrm{C}$, seguido de enfriamiento en un baño de agua con hielo a $5^{\circ} \mathrm{C}$ con el fin de someter las muestras a choque térmico (Morales et al., 2002; Miller y Miller, 2002). La temperatura se registró utilizando un Datalogger (TECPEL DTM-305). Luego se llevó las muestras a análisis sensorial.

\section{Extracción de la enzima PME}

Del jugo pasteurizado se extrajo la enzima PME según métodos reportados por Maca et al. (2012), Sentandreu et al. (2005), se diluyeron $400 \mu \mathrm{L}$ de jugo de uchuva en una solución de $1200 \mu \mathrm{L}$ de $\mathrm{NaCl}$ al $8,8 \%$ (p/v) Merck, junto con 0,008g de polivinilpirrolidona (PVPP) Sigma-Aldrich y se homogenizó la mezcla por 10min a 200rpm en una plancha de agitación magnética (THOMAS SCIENTIFIC), en seguida se centrifugó a 10.000rpm por 30min, en una centrífuga refrigerada (DYNAMICA VELOCITY 18R) y se recogió el sobrenadante (extracto enzimático) para medir la actividad enzimática de la PME.

\section{Medición de la actividad enzimática}

La actividad enzimática PME se analizó usando un espectrofotómetro UV-VIS (THERMO SCIENTIFIC GENESYS 10uv) según métodos reportados por Hagerman y Austin (1986), Maca et al. (2012), para la mezcla de la reacción se utilizó $1760 \mu \mathrm{L}$ de pectina cítrica al 0,5\%(p/v) Sigma-Aldrich (>85 \% esterificación), $530 \mu \mathrm{L}$ de azul de bromotimol al $0,01 \%(\mathrm{p} / \mathrm{v})$ CARLO ERBA en buffer fosfato de sodio $3 \mathrm{mM}$ Merck con un $\mathrm{pH}$ de $7,5,510 \mu \mathrm{L}$ de agua HPLC, y $70 \mu \mathrm{L}$ de extracto enzimático. Todos los reactivos se llevaron a $\mathrm{pH} 7,5$ con ayuda de $\mathrm{NaOH}$ al $1 \mathrm{~N}$ y $0,01 \mathrm{~N}$, cada análisis de actividad PME fue realizado por triplicado a $18^{\circ} \mathrm{C}$. El cambio de color de la mezcla de reacción se registró a una longitud de onda de $620 \mathrm{~nm}$. La actividad enzimática fue determinada de la parte lineal de la curva (inicio de la reacción enzimática), mediante regresión lineal, de donde se tomó al valor de la pendiente $(\mathrm{m})$ como la actividad PME $\left(\mathrm{A}_{0}\right)$ obtenida al 
evaluar la actividad PME en jugo sin pasteurizar y la actividad enzimática $\left(A_{t}\right)$ se tomó del valor de la pendiente $(\mathrm{m})$ obtenido al evaluar la actividad enzimática PME en jugo pasteurizado (Maca et al., 2012; Hagerman y Austin, 1986; Rudra et al., 2008). Los resultados se expresaron como el cambio en la densidad óptica por minuto ( $\triangle \mathrm{Abs} / \mathrm{min})$.

La actividad enzimática residual (AR), se calculó de la relación entre la actividad de la enzima después del tratamiento y la actividad que tiene sin tratamiento. Su cálculo se realizó utilizando la ecuación 1, reportada por Tiwari et al. (2009), Riener et al. (2009) y Carbonell et al. (2006) en términos de porcentaje:

$\% A R=\frac{A t}{A 0} \times 100$

En la ecn. (1), At es la Actividad de PME después del tratamiento térmico y $A_{0}$ es la Actividad de PME sin tratamiento térmico.

\section{Evaluación sensorial}

Se evaluó según la metodología descrita por Anzaldúa (1994), utilizando una prueba discriminativa triangular, una prueba descriptiva de ordenamiento y una prueba hedónica de preferencia, se emitió una valoración por 15 jueces entrenados, quienes compararon las diferentes muestras de jugo pasteurizadas con un jugo de uchuva sin pasteurizar (Navarro et al., 2011). La evaluación sensorial se realizó sobre acidez, color, olor y sabor. Para la prueba triangular se les presento a los jueces, tres muestras de jugo, de las cuales dos eran idénticas y se les solicito que identifiquen la muestra que era diferente, para la prueba de preferencia, se les presento a los jueces dos muestras de jugo y se les pidió que dijeran cuál de las dos preferían y para la prueba de ordenamiento se les entrego a los jueces 4 muestras de jugo para cada uno de los atributos sensoriales y se les solicito que ordenaran de menor a mayor intensidad cada atributo. Todas las muestras fueron codificadas con números aleatorios de 4 dígitos (Miller y Miller, 2002). La prueba triangular se analizó por medio de la tabla de interpretación estadística de Roessler et al. (1948), citada por Anzaldúa (1994), con un nivel de significancia del 5\%. La prueba de preferencia se analizó según la tabla de Roessler et al. (1956), citada por Anzaldúa (1994), con un nivel de significancia del 5\%. La prueba de ordenamiento se analizó mediante la prueba de comparación de rangos múltiples y el método de diferencia mínima significativa (HSD) de Tukey del paquete estadístico Statgraphics Centurion XVI.II (Miller y Miller, 2002).

\section{RESULTADOS Y DISCUSION}

Actividad enzimática inicial $\left(A_{0}\right)$ en jugo sin tratamiento térmico

Se obtuvo una $A_{0}=0,03403 \pm 0,019 \Delta A b s / m i n$, con ayuda de la ecuación (2).

$A b s=-0,03403 t+0,412 . \quad \mathrm{R}^{2}=0,9992$

En la ecn. (2), Abs es la absorbancia en $\mathrm{nm}$ y $\mathrm{t}$ es el tiempo en minutos

\section{Actividad enzimática residual PME (diseño factorial multinivel)}

Con ayuda del valor $A_{0}$ y los valores de $A_{t}$ resultantes de cada tratamiento térmico, Se cálculo los valores de actividad residual promedio en términos de porcentaje. La Fig. 1 muestra estos resultados.
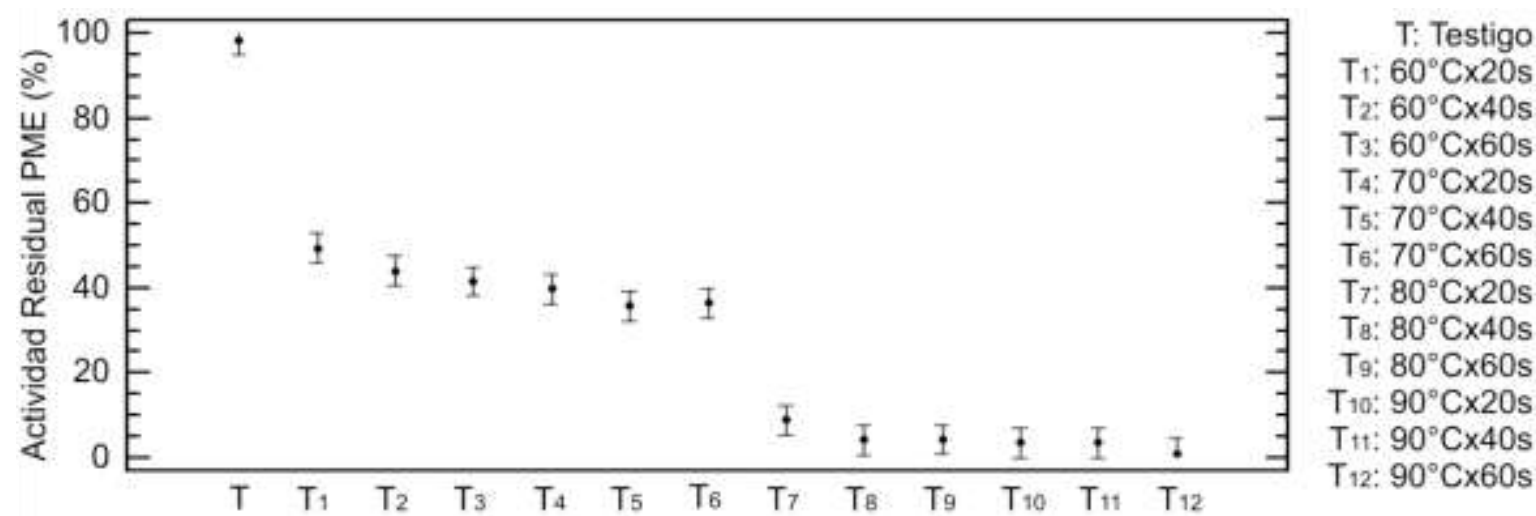

Fig. 1: Porcentaje de Actividad residual promedio PME en zumo de uchuva pasterizado. 
El incremento en la temperatura y en el tiempo de pasteurización, causa un efecto de disminución en la actividad residual PME, consiguiendo valores de actividad residual $\mathrm{PME} \leq 10 \%$, valores que permiten evitar la pérdida de consistencia y de homogeneidad del jugo (Croak y Corredig, 2006; Carbonell et al., 2006). Hay que mencionar, además que el análisis de varianza encontró que el cuadrado de la temperatura y el cuadrado del tiempo tienen un valor $\mathrm{P}<0,05$, lo que permite inferir que la pasteurización responde a una función cuadrática y que por lo tanto hay un punto óptimo de pasteurización. Así, el punto óptimo de pasteurización está en un rango desde los $70^{\circ} \mathrm{C}$ hasta los $80^{\circ} \mathrm{C}$, con actividades residuales de $38,87 \%$ y $8,96 \%$ (Fig. 1), para un tiempo de $20 \mathrm{~s}$, a partir de estos valores se plantea el diseño de composición central con superficie de respuesta.

\section{Actividad residual PME (diseño de composición central)}

La mejor interacción temperatura-tiempo del diseño de composición central continua siendo alrededor de los $80^{\circ} \mathrm{C}$ y con esto se confirma la tendencia presentada en el diseño factorial multinivel, en el que se obtiene una actividad residual PME $\leq 10 \%$, la Fig. 2 muestra estos datos.
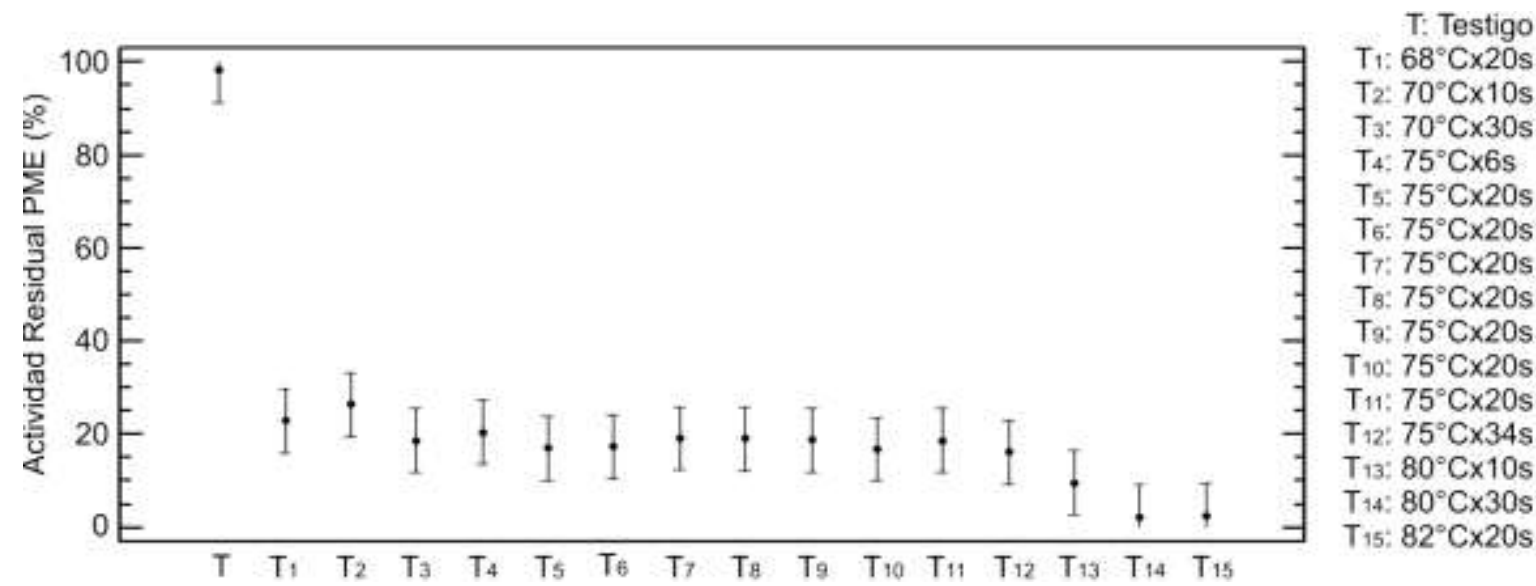

Fig. 2: Porcentaje de Actividad residual Promedio PME en zumo de uchuva pasterizado

En los tratamientos térmicos de este diseño continua prevaleciendo el cuadrado del tiempo (BB) y el cuadrado de la temperatura (AA) $(\mathrm{P}<0,05)$, lo que permite reafirmar que la reducción en la actividad residual PME en jugo de uchuva responde a una ecuación cuadrática y que por lo tanto hay un punto óptimo de pasteurización, para lo cual se optimiza los factores de proceso.

\section{OPTIMIZACIÓN DE LOS FACTORES DEL PROCESO}

Al optimizar los factores de proceso, temperatura y tiempo (Fig. 3, Tabla 1), se encontró que la mejor combinación es de $80{ }^{\circ} \mathrm{C} \times 10 \mathrm{~s}$, este parámetro permite una reducción $\geq 90 \%$ de la actividad enzimática inicial PME, que para el caso del jugo de uchuva resulto en $\% A R=9,32 \pm 0,57$, además se obtuvo la ecuación cuadrática (3), a la cual responde la actividad enzimática residual en función de la temperatura y tiempo, obtenida al aplicar el diseño de composición central:

$\% A R=-247,462+7,90962 \mathrm{~T}+3,17473 \mathrm{t}-0,057977 \mathrm{~T}^{2}-0,04695 \mathrm{Tt}+0,0088223 \mathrm{t}^{2}$

En la ecn. (3), AR es la actividad residual PME en \%, $\mathrm{T}$ es la temperatura en ${ }^{\circ} \mathrm{C}$ y $\mathrm{t}$ es el tiempo en segundos

Según Carbonell et al. (2006) y Maca et al. (2012) el nivel residual maximo enzimatico para jugos comerciales refrigerados es del $10 \%$, en el diseño factorial multinivel, se observo que a temperaturas de pasteurizacion entre los $60^{\circ} \mathrm{C}$ y los $80^{\circ} \mathrm{C}$, la PME se reduce desde el $52,12 \%$ hasta el $30,66 \%$ de actividad residual para tiempos de exposicion entre los $20 \mathrm{~s}$ y $60 \mathrm{~s}$, y desde los $80^{\circ} \mathrm{C}$ hasta los $90{ }^{\circ} \mathrm{C}$ y similares tiempos de exposicion, la actividad residual pasa del $8,96 \%$ al $1,13 \%$ lo que quiere decir que para jugo de uchuva la actividad enzimatica residual se reduce a niveles aceptables alrededor de los $80^{\circ} \mathrm{C}$. 


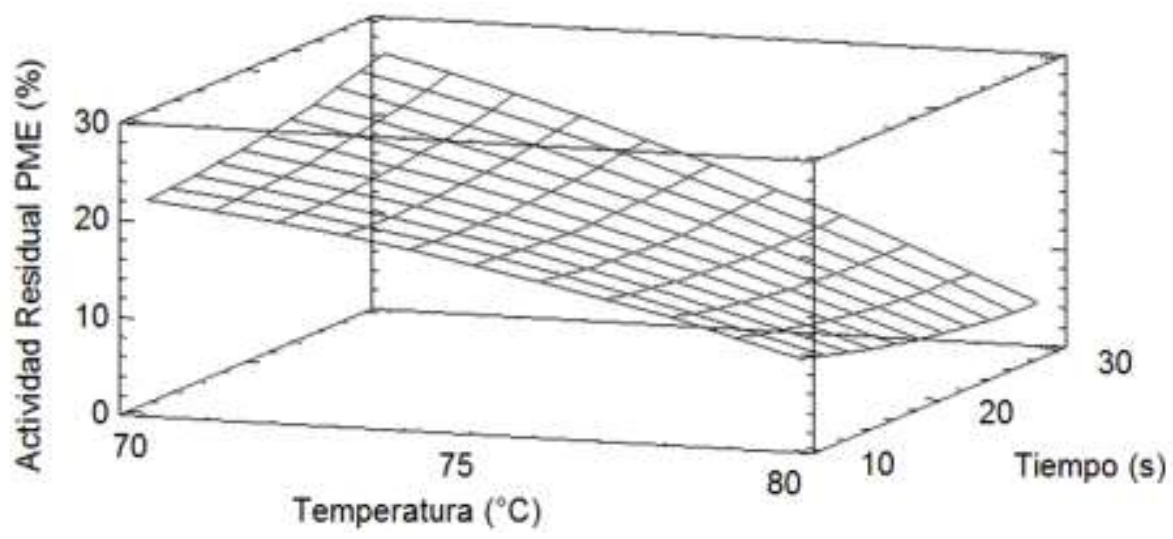

Fig. 3: Superficie de respuesta óptima para actividad residual PME

Tabla 1: Valor óptimo de los factores de temperatura y tiempo

\begin{tabular}{|l|l|l|l|}
\hline Factor & Bajo & Alto & Óptimo \\
\hline Temperatura $\left({ }^{\circ} \mathrm{C}\right)$ & 70 & 80 & 80 \\
\hline Tiempo $(\mathrm{s})$ & 10 & 30 & 10 \\
\hline
\end{tabular}

La pasteurización aplicada en el diseño de composición central, con temperaturas desde los $70^{\circ} \mathrm{C}$ hasta los $75{ }^{\circ} \mathrm{C}$ y tiempos de exposición entre los $10 \mathrm{~s}$ y los $30 \mathrm{~s}$, reduce la actividad residual desde el $22,54 \%$ hasta el $18,17 \%$ y de manera semejante al primer diseño aplicado, se observa que entre los $80^{\circ} \mathrm{C}$ y los $82{ }^{\circ} \mathrm{C}$, la actividad residual pasa del $9,32 \%$ hasta el $2,33 \%$ para tiempos de exposición entre los 10 s y $30 \mathrm{~s}$, todo esto parece confirmar que la actividad residual PME en jugo de uchuva, se ve notoriamente afectada a partir de los $80^{\circ} \mathrm{C}$, autores como Sentandreu et al. (2005), concluyen también que con una pasteurización de $85^{\circ} \mathrm{C}$ x $10 \mathrm{~s}$, se logra una actividad residual del 10\% para jugo de naranja y de forma similar Vivar et al. (2007), reportaron que en fruta de espino tratada térmicamente a temperaturas entre los $70^{\circ} \mathrm{C}$ y $80^{\circ} \mathrm{C}$, la perdida de actividad PME fue del $72,5 \%$ y $97,1 \%$ respectivamente y que a $80{ }^{\circ} \mathrm{C}$ durante 10 min la actividad enzimática residual fue completamente inactivada; lo dicho hasta aquí supone que la enzima fue desnaturalizada rápidamente con la aplicación de altas temperaturas.

Como consecuencia de optimizar los resultados del segundo diseño, se encontró que la mejor interacción temperatura-tiempo para inactivar PME en jugo de uchuva es de $80{ }^{\circ} \mathrm{C} \times 10 \mathrm{~s}$, con lo que la actividad residual PME se reduce hasta un 9,32\%, estudios realizados por Carbonell et al. (2006), tambien redujeron la actividad residual PME hasta el 6,5\% para una temperatura de $74{ }^{\circ} \mathrm{C}$ durante $10 \mathrm{~s}$, Siwach y Manish (2012) en jugo de Mosambi lograron una actividad residual PME del 10\% aplicando una pasteurización de $80{ }^{\circ} \mathrm{C}$ durante $10 \mathrm{~min}$, en síntesis la caída en la actividad PME se determina como una función de la temperatura y tiempo de pasteurización del Jugo, no obstante no toda la actividad PME se inactiva por pasteurización, sin embargo el mayor porcentaje de la fracción termoestable de la enzima se desnaturaliza en función estas condiciones utilizadas (Ingallinera et al., 2005) adicionalmente a esto Espachs et al. (2006), encontraron que a temperaturas mayores a $70^{\circ} \mathrm{C}$, la fracción termolábil de PME es rápidamente inactivada, mientras que la parte termoestable se inactivada gradualmente con el aumento de la temperatura y tiempo.

\section{EFECTOS DEL TRATAMIENTO TÉRMICO}

\section{Prueba triangular en jugo de uchuva}

De la prueba realizada, se obtuvo que de 14 jueces, 8 preferían el jugo sin tratamiento y 6 preferían el jugo pasteurizado a $80^{\circ} \mathrm{C} \times 10 \mathrm{~s}$, por lo tanto no se encontró diferencias significativas ( $\left.P>0,05\right)$ debido a que tan solo 8 prefieren el jugo sin tratamiento y se necesita que de 14 al menos 11 prefirieran la muestra sin pasteurizar para que haya diferencias significativas. Se analizó el grado aparente de diferencia tomando como referencia únicamente los 8 jueces que preferían la muestra diferente y se encontró que el grado está entre moderado y ligeramente moderado con respecto a la preferencia. Por lo tanto los jugos pasteurizados frente a un jugo fresco no se diferencian según la apreciación de los jueces (Anzaldúa, 1994).

\section{Prueba de preferencia entre jugo pasteurizado y sin pasteurizar}

De la prueba realizada se obtuvo que de 14 jueces, 7 preferían el jugo sin tratamiento y los 7 restantes prefieren el jugo pasteurizado a $80^{\circ} \mathrm{C} \times 10 \mathrm{~s}$, por ello no hay diferencias significativas $(P>0,05)$, debido a 
que se requiere que de 14 jueces al menos 11 prefieran la muestra sin pasteurizar para obtener diferencias significativas, acorde a esto, no hay diferencias significativas al $5 \%, 1 \%$ y $0,1 \%$ de significancia (Anzaldúa, 1994). De esta forma la aceptación entre un jugo natural y un jugo pasteurizado es igual, ya que los jurados evaluadores no detectaron diferencias significativas entre los jugos estudiados.

Prueba de ordenamiento para acidez, color, olor y sabor

Los resultados encontrados en la comparación de los cuatro tratamientos aplicados $\left(70{ }^{\circ} \mathrm{C} \mathrm{x} 10 \mathrm{~s}, 80^{\circ} \mathrm{C} \mathrm{x}\right.$ $10 \mathrm{~s}, 90^{\circ} \mathrm{C}$ x $10 \mathrm{~s}$ y sin pasteurizar) para las cuatro atributos sensoriales evaluados se muestran en la Fig. 4.

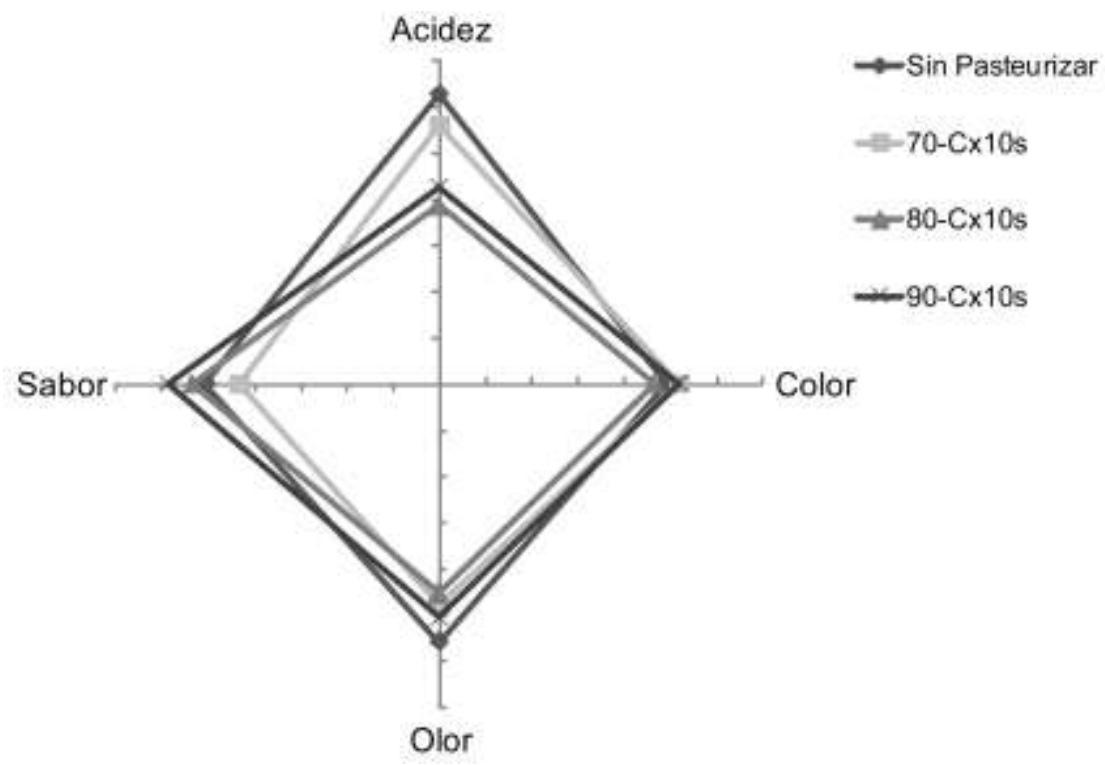

Fig. 4: Comparación de acidez, color, olor y sabor en 4 tratamientos aplicados a jugo de uchuva

Con un valor $P>0,05$, para los cuatro atributos del jugo de uchuva, se puede inferir que no existe una diferencia estadísticamente significativa para cada atributo comparado entre el jugo sin tratamiento y los jugos pasteurizados a $70{ }^{\circ} \mathrm{C}, 80^{\circ} \mathrm{C}$ y $90^{\circ} \mathrm{C}$ durante $10 \mathrm{~s}$, en resumen el jugo sin tratamiento frente a los jugos pasteurizados no se diferencia estadísticamente, autores como Hirsch et al. (2011) encontraron que incluso despues de aplicar una pasteurizacion a $85^{\circ} \mathrm{C}$ durante $10 \mathrm{~s}$ en jugo de naranja se logró mantener las cualidades organolépticas de un jugo fresco, de igual modo, los tratamientos aplicados al jugo de uchuva no afectan las propiedades organolépticas, sin embargo, hay que tener especial cuidado al aplicar una pasteurización severa, ya que es importante la conservación de las cualidades organolépticas y nutricionales del jugo las cuales se pueden ver afectadas por procesos térmicos prolongados (Sila et al., 2006; Tribess y Tadini, 2006).

\section{Seguimiento de la actividad enzimática}

El seguimiento de reactivación de la enzima PME se muestra en la Fig. 5.

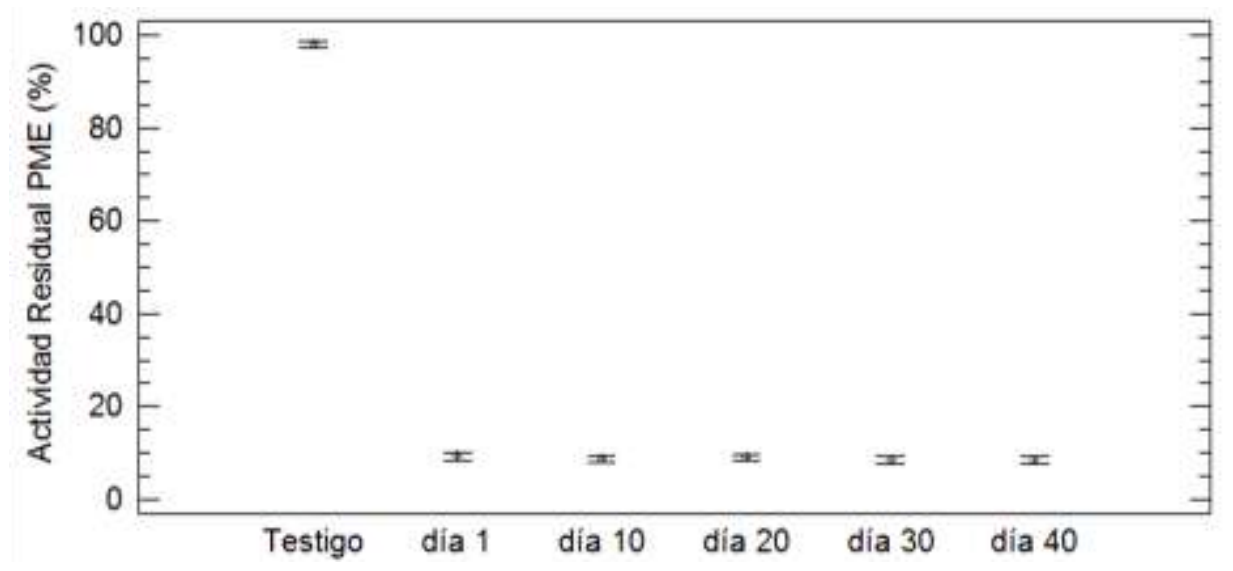

Fig. 5: Seguimiento actividad enzimática residual en jugo pasteurizado a $80^{\circ} \mathrm{C} \times 10 \mathrm{~s}$ 
La actividad enzimática no presenta reactivación hasta los 40 días de haber realizado la pasteurización en el jugo de uchuva, indicando que el tratamiento aplicado fue efectivo en la reducción de la actividad residual PME, ya que después de reducida la parte termoestable de la enzima es difícil que esta se reestructure nuevamente (Espachs et al., 2006).

\section{CONCLUSIONES}

De los resultados mostrados, se pueden obtener las siguientes conclusiones, sobre la Inactivación térmica de pectinmetilesterasa en jugo de uchuva: 1) Se logró determinar las condiciones óptimas de pasteurización del jugo de uchuva $\left(80^{\circ} \mathrm{C} \times 10 \mathrm{~s}\right)$ mediante las cuales se alcanzó una actividad enzimática residual permisible de 9,32\%, no obstante, la separación de fases continuó prevaleciendo en el jugo pasteurizado, mostrando que la actividad enzimática de la pectinmetilesterasa no está relacionada directamente con la separación de fases del jugo de uchuva. 2) Se optimizo la pasteurización con ayuda de tubos capilares para microhematocrito los cuales cumplieron con las condiciones dadas, permitiendo minimizar el tiempo de subida de la temperatura al interior del jugo lo cual favoreció en gran medida al proceso térmico. 3) El método térmico aplicado no provoco cambios significativos en las características sensoriales del producto pasteurizado, sin embargo, se hace necesario que algunos parámetros como el color se evalúen mediante equipos electrónicos, a fin de reafirmar los resultados obtenidos.

\section{AGRADECIMIENTOS}

A la Vicerrectoría de Investigaciones, Postgrados y Relaciones Internacionales VIPRI de la Universidad de Nariño, Colombia, por la financiación de esta investigación.

\section{REFERENCIAS}

Anthon, G., Y. Sekine, N. Watanabe y D. Barrett, Thermal inactivation of pectin methylesterase, polygalacturonase, and peroxidase in tomato juice, Journal of Agricultural and Food Chemistry: 50 (21), 6153-6159 (2002).

Anzaldúa, M. A., La evaluación sensorial de los alimentos en la teoría y la práctica, 78-92, Editorial Acribia, S.A, Zaragosa, España (1994).

Baker, R. A. y R. G. Cameron, Clouds of citrus juices and juice drinks, Food Technology: 53 (1), 64-69 (1999).

Basak, K. y H. S. Ramaswamy, Ultra high pressure treatment of orange juice: a kinetic study on inactivation of pectin methyl esterase, Food Research International: 29 (7), 601-607 (1996).

Carbonell, J. V., P. Contreras, L. Carbonell, J. L. Navarro, Pectin methylesterase activity in juices from mandarins, oranges and hybrids, European Food Research and Technology: 222 (1-2), 83-87 (2006).

Cinquanta, L. y otros tres autores, Effect on Orange Juice of Batch Pasteurization in an Improved PilotScaleMicrowave Oven, Journal of Food Science: 75 (1), E46-E50 (2010).

Croak, S. y M. Corredig, The role of pectin in orange juice stabilization: Effect of pectin methylesterase and pectinase activity on the size of cloud particles, Food Hydrocolloids: 20 (7), 961-965 (2006).

Espachs, A., A. Van Loey, M. Hendrickx, O. Martín-Belloso, Inactivation of plant pectin methylesterase by thermal or high intensity pulsed electric field treatments, Innovative Food Science and Emerging Technologies: 7 (1-2), 40-48 (2006)

Fischer, G., D. Miranda, W. Piedrahita, J. Romero, Avances en cultivo poscosecha y exportación de la uchuva (Physalis en peruviana) (2005). http://www.asohofrucol.com.co/archivos/biblioteca/biblioteca_23_Avances\%20cultivo\%20uchuva.pdf. Acceso: 6 de Septiembre (2013).

García, H., B. Brito y M. C. García, Desarrollo tecnológico para el fortalecimiento del manejo postcosecha de frutales exóticos exportables de interés para los países andinos:Uchuva (Physalis peruviana L.), granadilla (Passiflora ligulars L.), y tomate de árbol (Cyphomandra betacea (Cav) S.) (2008). http://www.fontagro.org/sites/default/files/stecnico/final_infotec_03_14.pdf. Acceso: 1 de Agosto (2013). 
Hagerman, A. E. y P. J. Austin, Continuous spectrophotometric assay for plant pectin methyl esterase, Journal of Agricultural and Food Chemistry: 34 (3), 440-444 (1986).

Hirsch, A., A. Knauss, R. Carle y S. Neidhart, Impact of minimal heat-processing on pectin methylesterase and peroxidase activity in freshly squeezed Citrus juices, European Food Research and Technology: 232 (1), 71-81 (2011).

Ingallinera, B., R. Barbagallo, G. Spagna, R. Palmeri y A. Todaro, Effects of thermal treatments on pectinesterase activity determined in blood oranges juices, Enzime and Microbial Technology: 36 (2-3), 258263 (2005).

Maca, M., O. Osorio y D. Mejía, Inactivación Térmica de Pectinmetilesterasa en Tomate de Árbol (Solanum betaceum), Información Tecnológica: 24 (3), 41-50 (2013).

Mendoza, J., A. Rodríguez, P. Millán, Caracterización Físico Química de la Uchuva (Physalis peruviana) en la Región de Silvia Cauca, Biotecnología en el Sector Agropecuario y Agroindustrial: 10 (2), 188-196 (2012).

Miller, N. J. y J. C. Miller, Estadística y Quimiometría para Química Analítica, 4a Edición, PEARSON EDUCACIÓN. S. A., Madrid, España (2002).

Montgomery, D. C. y G. C. Runger, Applied Statistics and Probability for Engineers, Third Edition, John Wiley \& Sons, Inc., New York, United States of America (2003).

Morales, B. E., V. Chandia y L. Cisneros Z, Thermal Inactivation Kinetics of Peroxidase and Lipoxygenase from Broccoli, Green Asparagus and Carrots, Journal of Food Science: 67 (1), 146 - 154 (2002).

Navarro, P., A. J. Melendez, F. Heredia, J. A. Gabaldon, A. A. Carbonell, A. Soler y A. J. Perez-Lopez, Effects of $\beta$-cyclodextrin addition and farming type on vitamin $C$, antioxidant activity, carotenoids profile, and sensory analysis in pasteurised orange juices, International Journal of Food Science \& Technology: 46 (10), 2182-2190 (2011).

NTC 4580, Instituto Colombiano de Normas Técnicas y Certificación (ICONTEC), Frutas Frescas, Uchuva Especificaciones, Bogotá D.C., Colombia (1999).

Osorio, O., N. Martínez, G. Moraga y J. V. Carbonell, Effect of Thermal Treatment on Enzymatic Activity and Rheological and Sensory Properties of Strawberry Purees, Food Science and Technology International: 14 (5), 103-108 (2008).

Raviyan, P., Z. Zhang y H. Feng, Ultrasonication for tomato pectinmethylesterase inactivation: effect of cavitation intensity and temperature on inactivation, Journal of Food Engineering: 70 (2), 189-196 (2005).

Riener, J., F. Noci, D. A. Cronin, D. J. Morgan, y J. G. Lyng, Combined effect of temperature and pulsed electric fields on pectin methyl esterase inactivation in red grapefruit juice (Citrus paradisi), European Food Research and Technology: 228 (3), 373-379 (2009).

Rudra, G., U. S Shivhare y S. Basu, Termal inactivation kinetics of peroxidasa in mint leaves, Journal of Food Engineering: 85 (1), 147-153 (2008).

Sentandreu, E., L. Carbonell, J. V. Carbonell y L. Izquierdo, Effects of Heat Treatment Conditions on Fresh Taste and on Pectinmethylesterase Activity of Chilled Mandarin and Orange Juices, Food Science and Technology International: 11 (3), 217-222, (2005).

Sila, D. N., C. Smout, F. Elliot, A. Van Loey y M. Hendrickx, Non-enzymatic depolymerization of carrot pectin: Toward a better understanding of carrot texture during thermal processing, Journal of Food Science: 71 (1), E1-E9 (2006).

Siwach, R. y K. Manish, Comparative study of thermosonication and thermal treatments on pectin methylesterase inactivation in mosambi juice, Asian Journal of Dairying \& Foods Research: 31 (4), 290 - 296 (2012). 
Tiwari, B. K., K. Muthukumarappan, C. P. O'donnell y P. J. Cullen, Inactivation Kinetics of pectin methylesterase and cloud retention in sonicated orange juice, Innovative Food Science \& Emerging Technologies: 10 (2), 166-171 (2009).

Tribess, T. B. y C. C. Tadini, Inactivation kinetics of pectin methylesterase in orange juice as a function of $\mathrm{pH}$ and temperature/time process conditions, Journal of the Science of Food and Agriculture: 86 (9), 1328-1335 (2006).

Vila, L. R., Caracterización fisicoquímica del membrillo japonés y desarrollo fisiológico y conservación frigorífica, Tesis de Doctorado, Departamento de Tecnología de Alimentos, Nutrición y Bromatología, Universidad de Murcia, Murcia-España (2006).

Vivar, V. M., J. Montoya, G. Calva, E. G. Ramos, Extraction, thermal stability and kinetic behavior of pectinmethylesterase from hawthorn (Crataegus pubescens) fruit, LWT-Food Science and Technology: 40 (2), 278-284, (2007).

Willats, W., L. Mccartrney, W. Mackie, J. Knox, Pectin: cell biology and prospects for functional analysis, Plant Molecular Biology: 47, 9-27 (2001). 